\title{
Simple electromagnetic spider web placement and use method
}

\author{
Min JIANG
}

\begin{abstract}
Ningbo Polytechnic 315800 Ningbo 315800, China
KEYWORD :Earthquake prediction; Magnetic switch module; GSM; wireless transmitting and receiving module; Electromagnetic GPRS network spider nets; The Tanlu fault zone
\end{abstract}

\begin{abstract}
We design the electromagnetic spider web network in the country from the first spider placed the Tanlu fault zone. This fault through the place, a large population, economic development, Hefei is the first place, Shenyang will be our second point to place the location. Mr. Li Siguang once noted: in the Chinese status of the Tanlu fault zone, pointed out that the Tanlu fault zone is to focus on the earthquake fortification; because of its geographical location special led to the current understanding of it's new tectonic activity is relatively small, according to the historical experience of the possibility of future fracture of the devastating earthquake is very great big. Simple electromagnetic spider web from this area, and found that the electromagnetic anomaly after a large number of placed in order to determine the earthquake three elements.
\end{abstract}

\section{1,INTRODUCTION}

The simple electromagnetic spider web is composed of the electric field emission and the electric field receiver circuit when the anomaly of the magnetic field is abnormal. Here is the design of when the magnetic anomaly detection space electric field whether normal of the electromagnetic spider web is practical and reliable, the main is the power saving, don't need deep in the interior of the earth as long as placed in empty space, the normal use of time is two years. Can be used to detect the electromagnetic anomaly before the earthquake, the earthquake prediction control system is very simple. After many years of testing, the magnetic field electronic compass part of the general connection LED four times, each time the opening of 10 seconds, normally closed (5 seconds), and then on (10 seconds), off (5 seconds). If the detected magnetic anomaly on (27MHz and $49 \mathrm{MHz}$ and $315 \mathrm{MHz}$ ) field emission, the host includes receiving a cycle detection circuit, GSM alarm made impending earthquake predictor will be before the earthquake electromagnetic parameters conversion for digital signal of electrical parameters, the GSM remote switch alarm. Both theory and practice can prove that it can not only be used to predict electromagnetic field anomaly before earthquake, but also can be used to determine the location of the epicenter. Perception of electromagnetic field changes within a few hours to a few days before the earthquake. Can know in time that the earthquake is about to happen, and determine the location of the epicenter.

\section{2, ELECTRIC PLACEMENT METHOD:}

(Electric field) placement method in detail: two point electromagnetic spider web. A series of electrodynamics equations Maxwell equations which is based the theoretical basis of the design and deployment of transmitter. Electronic stopwatch and PLC is the core of the design of the receiving system, the long term timing transmitting circuit. Actual installation requires only two groups. The other half of the receiving area is usually in the non receiving area. Long distance alarm circuit does not start when the normal electromagnetic GSM, abnormal electromagnetic generation on the remote automatic alarm, alarm and self lock after the lifting of the remote control to launch the "output off" command. One charge can be more than 200 times the alarm, very convenient. The electromagnetic spider web that we designed is most sensitive in the $27 \mathrm{Mhz} 49 \mathrm{Mhz}$ section. Scan every hour in 5 seconds automatically from low frequency to high frequency scan, respectively in the remote receiver can respectively receive signals, a plurality of electromagnetic spider groups, combined with the GPRS remote monitoring device can be analyzed accurately in time and location of the earthquake epicenter of the earthquake level. Four section 5 battery is expected to use life in $1 \sim 3$ years, generally do not require manual intervention. Ordinary home, as long as the choice of 2 or more can be early warning. The remote automatic alarm is generated when the abnormal 
electromagnetic is produced, and the alarm is self locked, and the output is transmitted by the remote control.

\section{3, MAGNETIC PLACEMENT METHOD:}

(Magnetic field part) is the host and the sensor placement method (E-Compass) should be in the distance of about $50 \mathrm{~cm}$, to prevent mutual interference; within the host cell and battery powered timer should generally be replaced every year, for the 5 three AA batteries; electromagnetic testing method is to use simple Web quickly across the ordinary mobile phone sensor above, the host will send out alarm sound; indicator lamps for general LED on four times per minute, each lit for 10 seconds, normal dark light (5 seconds) show that the circuit works normally; if the alarm once every 1 hours interval, indicating the earthquake will in the next 1 hours, every 10 minutes interval the alarm time, that the earthquake will happen in the next 10 minutes. Electromagnetic spider net (magnetic field) should be damp proof, anti high temperature, anti vibration, anti movement.

\section{4,THE WEB PLACEMENT STRATEGY:}

Go Game: The use of electromagnetic spider network prediction of earthquake is like with the earthquake before the abnormal electromagnetic field play Go online, the layout of the stage of the electromagnetic spider web is generally placed in each of the city. Spacing to be coherent. To have a sense of rhythm and rhythm, each instrument is like a fresh beat of life. From beginning to end the abnormal electromagnetic field changes before the earthquake to maintain an unusually keen sense of ability. Once the abnormal signal must be hard for. Trying not to lose control of the signal. When the direct prediction fails when, according to the time and place of the accumulation of electromagnetic anomalies appeared with a variety of mathematical methods retreat road interference electromagnetic anomalies threat to knock sticky candy way to small victories. No matter self repair. You tighten the fist, in fact, in order to better expose the seismic anomalies before the earthquake law. No, it can always feel comfortable, Ever fount inspiration. Go to what is, what is lost, get lost, lost in something, no one thing is true, but. This place must be placed. This is the key to the momentum conversion, the slightest slack. The key is likely to be so a few points, can not relax. When receiving the apparatus to be delicate tactics. Crude and careless, will make your own layout in the crowning calamity not careful. Stability is more important than anything. The quasi abnormal electromagnetic field pulse, each placed the best point to the electromagnetic anomalies before the earthquake to the mainpoint, or will become abnormal electromagnetic field shield door side. Of course, this requires a kind of advance of the imagination. Location: on the one hand can be revealed before the Earthquake abnormal electromagnetic field, the opponent cannot quickly settle; on the other hand can consolidate their place. The land must go to, you cannot regret it. To feel at any time the abnormal electromagnetic field to occupy different positions on the pressure of their soldiers. According to the changing situation, don't get bogged down in the small place. Cloth out of the potential to be generous, stretch angry. Never dull. No good if the receiving means appear abnormal electromagnetic field, first put down. In order to find the maximum net, wait. To choose the most conducive to their own place and the abnormal electromagnetic field, a small place do not have to do too much entanglement. To allow the abnormal electromagnetic field to follow their own rhythm. Selection of convenient traffic in the city should be placed on the premise of the whole network is thick. Each electromagnetic spider web to take care of each other, the formation of an organic whole. If there is only one instrument to Vicenza, this potential is extremely powerful. Otherwise, it is a disadvantage. Can not detect all three elements of the earthquake.

\section{5, THE METHOD USED TO DETERMINE THE LOCATION OF THE EPICENTER :}

The electromagnetic spider web is a device that will be placed immediately after the appearance of the abnormal electromagnetic signal. The basic methods are placed in single line battle array. A plurality of electromagnetic web line such as Hydra array operation computation, like a python attack. The other two methods such as cavalry, placing most mobile, is the so called dragon water array. Liuzhou array consists of multiple electromagnetic water spider two road,like 
two dragons of the place. At the same time in the middle part put forward, forming the so called world powers array. Then two retracement, forming the so called four gate array fallback. If a plurality of electromagnetic webs interspersed with each other, and become Wuhu sheep array. Wuhu sheep array is the strongest range front found before the earthquake electromagnetic anomalies, arranged in 5 words written in Greek in the lineup, but with the symbol of victory in the words of $\mathrm{V}$ the same type, not war had been occupied in front of absolute advantage. Then according to the six butyl arranged into six butyl array. If half the entire matrix pull into line (can change), half as four door general electromagnetic array fallback, spider web consisting of the big dipper. At the same time around a circle, according to the gossip lineup, leaving eight of exports, into a square, which is the electromagnetic spider web eight golden array. You can also press the ninth order, each grid will gradually as a whole, interspersed with each other to wear, which is the electromagnetic spider nets nine word serial array. Most place becomes ambush array. Multi channel array calculation to ambush abnormal electromagnetic signals, calculate the most vulnerable or most powerful abnormal signal is the epicenter location using a variety of digital simulation model.

\section{6,THE TANLU FAULT ZONE:}

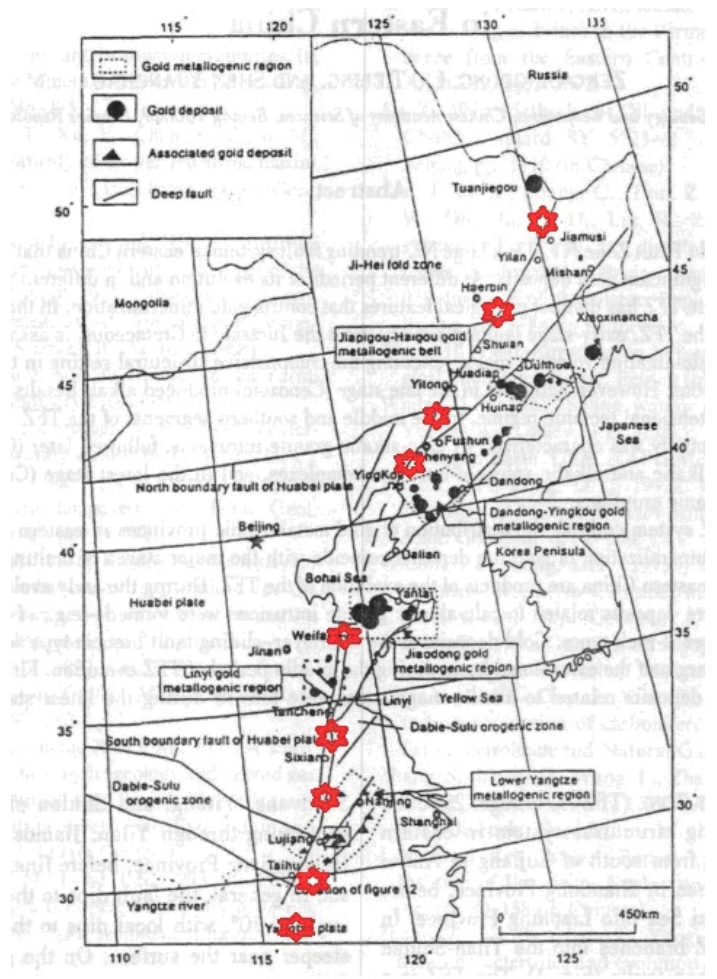

Fig.1 (*)TFZ placed electromagnetic spider web

Already in the Tanlu fault zone placed electromagnetic spider web in fig.1. From Shenyang to Shandong Tancheng Anhui Lujiang zone of the Tanlu fault. Across Liaoning, Bohai, Shandong, jiangsu. The Tanlu fault zone controlled the tectonic framework of the eastern china. So, study on the Tanlu fault zone, not only has profound academic significance, but also has close practical value. The Tanlu fault zone formation time recognize there are large differences. There are two main views: one is that the formation of the 600 million years ago in the Cambrian, the other is that more than one hundred million years ago in the Mesozoic era. There is also a view that the gradual increase in the development of the Paleozoic era. The Tanlu fault zone was found that its formation and evolution can be summarized as the trilogy. It formed in one hundred million years ago in the late Jurassic and left a huge horizontal movement; about one hundred million years ago to 45 million years (the geological age of the late Cretaceous to early three century), to open the main movement, formed in this basin; since in extrusion movement. Its activities are controlled by the movement of the plates in the Pacific Ocean. 
Including from Suqian to Liaoning, Tieling, Hebei, Shandong, Jiangsu and other provinces in most or part of the region. Is a strong seismic activity zone in the eastern part of China. The Tanlu fault in Jiangsu, Shandong border intersection. Since 1990 has been listed for seismic risk in the key monitoring region, State Seismological Bureau, the Tanlu fault belt in strong extrusion and both dextral fault segments. Because of its great stress, the shear stress is also large, it is easy to accumulate the energy of large earthquake, and it is difficult to release in the form of medium and small earthquakes, so the seismic intensity of the segment is large, and the frequency is low.

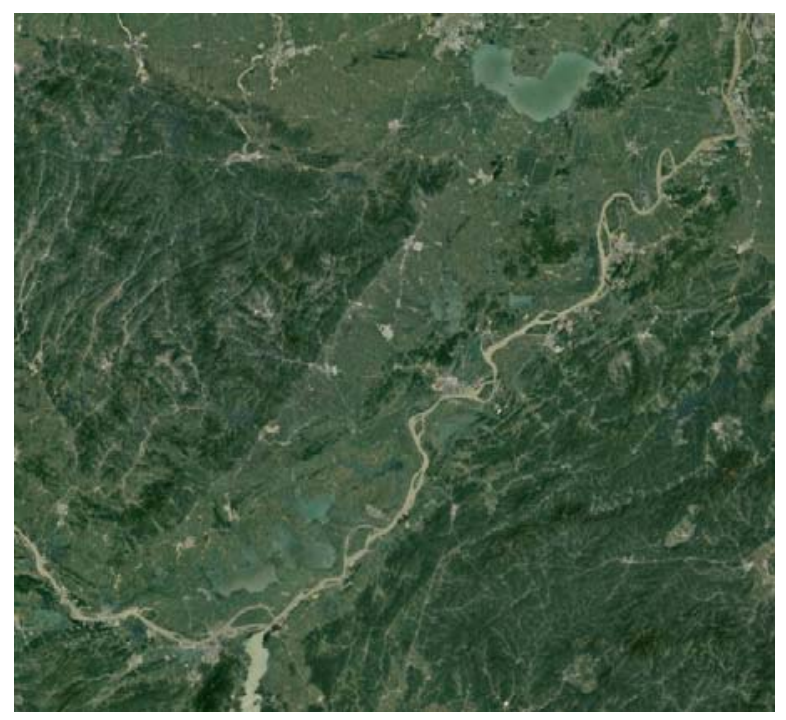

Fig.2 Part of the Tanlu fault zone

The Tanlu fault zone is a large fault, Tan City, Shandong and Anhui Lujiang first word naming, in this fracture is possible occurrence of an earthquake. This fault by Wang tie Feidong Cuozhen town to Lujiang, Tongcheng, Qianshan to wipe the Dabie Mountains and the eastern margin. History, the biggest earthquake in Tanlu seismic belt occurred in 1668, the magnitude was 8 . The earthquake affected more than half of China, also affected North Korea and Japan, also caused 7 to 8 degrees in Hefei damage, at that time, the walls of Hefei have been destroyed."

The earthquake is immune. Under normal circumstances, more than 7 earthquake, the accumulation of time need 20003000 years, the stress and energy release of light, and the latter takes a long time. The Tanlu seismic belt of Anhui section has been "immune", the possibility of future is earthquake. The Tanlu fault zone is in East Asia, a series of North East to giant fault system in the trunk of a fracture zone, in the territory of China extends more than 2400 kilometers, cut through the eastern China of different tectonic units, the grand scale, complex structure. The joint belt of the crustal fault block movement, which is the usual belt of the geophysical field and the deep source magmatic activity zone. It was formed in the mesoproterozoic. The Tanlu fault and exists not only between the Tancheng Lujiang, South to Wuxue, Hubei Province, the north shore of the Yangtze River, North and east to the Anhui Province Susong, hill, Lujiang, Jiashan, Jiangsu Sihong County, Suqian, Shandong Tancheng, Yishui, Weifang, into the Bohai Sea, and Liaodong Peninsula through three provinces in the northeast of China to Russia. Within the territory of China for up to $2400 \mathrm{~km}$, a few dozen to $200 \mathrm{~km}$, the overall trend of the North East 1020 degrees. The Tanlu fault slip rate of the current level of about $2.3 \mathrm{~mm} /$ year.

Already in the Tanlu fault zone placed electromagnetic spider web in figure 1. The Tanlu fault zone in Chinese territory of more than 2 thousand kilometers long, from Anhui, Jiangsu, Shandong, into Bohai, after landing in Liaoning, entered the northeast, divided into two teams (the other one is divided into three branches), and then to the north, into russia. It was formed in 200 million years ago Triassic, at that time the dinosaurs began to appear. Its formation should be closely related to the collision of the North China block and the Southern China block. You can see from Google maps for the north, Southern China two plots collision forms a towering mountains in the middle, is the Qinling Mountains Dabie mountains. Because millions of years of weathering and erosion in the charm. After the formation of this fault, it has experienced a complicated evolution. The first place in the city of Hefei and then put down from Shenyang early layout, must be carefully selected, 
Google map visible there is a very obvious line of Tanlu fault zone controlled the tectonic framework of the eastern china. With the electromagnetic placed spider webs in the Tanlu fault, has convenient detection to the practical research value of abnormal electromagnetic field. The Tanlu Fault Zone grand scale, complex structure. The joint belt of the crustal fault block movement, which is the usual belt of the geophysical field and the deep source magmatic activity zone. In the formation of middle proterozoic. Through the multi period structure. The active fault zone, which is mainly based on the right hand and the right hand, is also a seismic activity belt with distinct segment and varying degrees of activity.

The Tanlu fault zone is the East Asian continent on a series of NNE a giant fault system in the main fault zone, extending more than 2 thousand and 400 kilometers in China, cut through the eastern China in different tectonic units, grand scale, complex structure. The joint belt of the crustal fault block movement, which is the usual belt of the geophysical field and the deep source magmatic activity zone. In the formation of middle proterozoic. Through the multi period structure. It is not only a "longevity" to shearing movement deep fracture zone, and it is a new sport recently still inherit the structure, to push the right inverse of active fault zone, but also has a significant seismic activity, with varying degrees of activity section. We design the network from the first electromagnetic spider Tanlu fault zone start. If found to have abnormal electromagnetic signals coming from the remote transmission, immediately start the electromagnetic spider nets. With an interval of 100 square kilometers of distance to place the electromagnetic spider web. Calculation of abnormal electromagnetic field signal by inducing array multi channel to calculate the ambush, the most vulnerable or most powerful abnormal signal is the epicenter location using a variety of digital simulation model.

\section{7、SUMMARY:}

Electromagnetic spider is not deep to learn. Log Spiral gradient eight point placement of temperature. As the weaving speed rotation magnetic field detection do not be afraid of the pain. Plate moving divergence the change of the electric field to take care of. Identify the road forward and Germany and Japan dispute low high. Unique design carefully installed carefully debug bold. Life is short to strive for the benefit of the public light fame and fortune. He predicted that the prime minister wishes to realized.

\section{8、REFERENCE:}

1、Jiang Min. Multi point timing control circuit [J]. Electric world fourth, 1992 (4): 42

2、 Jiang Min. Sound and light indicator of electronic compass [J].Electric world, second 1996 (2): 33

3、Jiang Min. Earthquake prediction micro integrated measurement recorder [J] Technology Innovation Herald 2010, (29): 2223

4、Min Jiang,2013.11 How to weave the electromagnetic spider web to predict earthquakes, 2013 3rd International Conference on Education and Education Management(EEM 2013), p546 551

5、 Min Jiang,2014.1 Electromagnetic spider web Application in earthquake prediction, International Core Journal of Scientific Research \& Engineering Index,p111 114.

6、 Min Jiang,2014.11 The Group of Electromagnetic spider web, Economic, Business Management and Education Innovation(EBMEI 2014),p287 291.

7、 Min Jiang,2014.12 Low Power Consumption Electromagnetic spider web, 2014 4th International Conference on Education and Education Management(EEM 2014), p74 78.

8、 Min Jiang, Ping Yao Wang 2015.9 Simple space electric field and earth magnetic anomaly remote alarm system,2015 International Conference on Frontiers of Manufacturing Science and Measuring Technology, p452 458.

9、Min Jiang ,2016.4 Application of 3 kinds of practical electromagnetic spiders in electromagnetic spider web. ICEICE 2016 02083. MATEC Web of Conferences 44.

10、Min Jiang ,2016.9 Easily magnetic anomalies earthquake prediction. MMME 050910. 